\title{
Role for rodent Smc6 in pericentromeric heterochromatin domains during spermatogonial differentiation and meiosis
}

\author{
DE Verver ${ }^{1}$, AMM van Pelt ${ }^{1}$, S Repping ${ }^{1}$ and G Hamer ${ }^{*}, 1$
}

Chromatin structure and function are for a large part determined by the six members of the structural maintenance of chromosomes (SMC) protein family, which form three heterodimeric complexes: Smc1/3 (cohesin), Smc2/4 (condensin) and Smc5/6. Each complex has distinct and important roles in chromatin dynamics, gene expression and differentiation. In yeast and Drosophila, Smc6 is involved in recombinational repair, restarting collapsed replication forks and prevention of recombination in repetitive sequences such as rDNA and pericentromeric heterochromatin. Although such DNA damage control mechanisms, as well as highly dynamic changes in chromatin composition and function, are essential for gametogenesis, knowledge on Smc6 function in mammalian systems is limited. We therefore have investigated the role of Smc6 during mammalian spermatogonial differentiation, meiosis and subsequent spermiogenesis. We found that, during mouse spermatogenesis, Smc6 functions as part of meiotic pericentromeric heterochromatin domains that are initiated when differentiating spermatogonia become irreversibly committed toward meiosis. To our knowledge, we are the first to provide insight into how commitment toward meiosis alters chromatin structure and dynamics, thereby setting apart differentiating spermatogonia from the undifferentiated spermatogonia, including the spermatogonial stem cells. Interestingly, Smc6 is not essential for spermatogonial mitosis, whereas Smc6-negative meiotic cells appear unable to finish their first meiotic division. Importantly, during meiosis, we find that DNA repair or recombination sites, marked by $\gamma \mathrm{H} 2 \mathrm{AX}$ or Rad51 respectively, do not co-localize with the pericentromeric heterochromatin domains where Smc6 is located. Considering the repetitive nature of these domains and that Smc6 has been previously shown to prevent recombination in repetitive sequences, we hypothesize that Smc6 has a role in the prevention of aberrant recombination events between pericentromeric regions during the first meiotic prophase that would otherwise cause chromosomal aberrations leading to apoptosis, meiotic arrest or aneuploidies.

Cell Death and Disease (2013) 4, e749; doi:10.1038/cddis.2013.269; published online 1 August 2013

Subject Category: Cancer

Chromatin structure and function are for a large part determined by the structural maintenance of chromosomes (SMC) protein family. In association with several other nonSMC subunits, these proteins form three types of ring-like complexes: cohesin (Smc1/3), condensin (Smc2/4) and the Smc5/6 complex, all of which have the capacity to hold two double-stranded DNA strands together. ${ }^{1,2}$ Cohesin is required for sister chromatid cohesion and properly timed segregation during mitosis. Furthermore, also by holding sister chromatids together, cohesin is essential for DNA damage repair by homologous recombination during the $S$ and $G 2$ phases of the cell cycle. ${ }^{1-4}$ Condensin's main function is to form loops within a single chromosome, thereby causing condensation of the chromosomes required for the mitotic divisions. ${ }^{5}$ In addition, both cohesin and condensin are thought to have roles in gene expression. ${ }^{6}$ Cohesin is known to contribute to the gene regulatory functions of CTCF (CCCTC-binding factor), an enhancer-blocking insulator protein, by altering chromosomal conformation in order to influence the interaction between promoter and enhancer elements. ${ }^{7-10}$ Moreover, it has been proposed that dynamic placement of cohesin complexes by pluripotency transcription factors, contributes to a chromosome organization that maintains pluripotency of embryonic stem cells. ${ }^{11}$

The Smc5/6 complex was first described in yeast cells to be involved in the DNA damage response process by promoting sister chromatid recombination and recruiting cohesin to double-strand break (DSB) sites. ${ }^{4,12-14}$ Similarly, it has been shown that the yeast Smc5/6 complex is required to resolve meiotic recombination intermediates and that abrogation of the complex leads to unresolved linkages between the meiotic chromosomes. ${ }^{15-17}$ Although DNA damage repair has long

\footnotetext{
${ }^{1}$ Center for Reproductive Medicine, Women's and Children's Hospital, Academic Medical Center, University of Amsterdam, Amsterdam, The Netherlands ${ }^{*}$ Corresponding author: G Hamer, Center for Reproductive Medicine, Women's and Children's Hospital, Academic Medical Center, University of Amsterdam, Meibergdreef 9, Q3.119, 1105 AZ Amsterdam, The Netherlands. Tel: + 3120 5661851; Fax: + 3120 5669044; E-mail: g.hamer@amc.uva.nl

Keywords: structural maintenance of chromosomes-6; pericentromeric heterochromatin domains; spermatogonial differentiation; meiosis; meiotic recombination Abbreviations: $\gamma \mathrm{H} 2 \mathrm{AX}$, gamma-histone 2AX; ALT, alternative lengthening of telomeres; CREST, calcinosis, Raynaud's syndrome, esophageal motility, sclerodactyly, telangiectasias; CTCF, CCCTC-binding factor; CTCFL, CTCF-like; DAPI, 4'6-diamidino-2-phenylindole -2HCl; DSB, DNA double-strand break; MSCI, meiotic sex chromosome inactivation; MSUC, meiotic silencing of unsynapsed chromosomes; SCP3, synaptonemal complex protein 3; SMC, Structural maintenance of chromosomes; STAG3, stromal antigen 3; VAD, vitamin A-deficient

Received 09.4.13; revised 14.6.13; accepted 19.6.13; Edited by A Stephanou
} 
been proposed to be its main function, in many mammalian cancer cells lacking telomerase activity, Smc5/6 is also involved in a process called alternative lengthening of telomeres $(\mathrm{ALT})$ by promoting intra-telomeric recombination. ${ }^{14,18-20}$ Moreover, apart from facilitating recombination, yeast Smc5/6 has also been found to provide structural organization and topological relief to replication forks in mitotically dividing cells $s^{4,21-28}$ and could perhaps even be involved in gene silencing. ${ }^{29-32}$

In contrast, in heterochromatic regions containing densely packed, highly repetitive DNA sequences such as rDNA and the pericentromeric regions, Smc5/6 maintains genomic stability by suppressing aberrant intra-chromosomal recombination between these repetitive sequences. ${ }^{14,26,33,34}$ Additionally, Smc6 is required for repair of DSBs that occur in these heterochromatic regions ${ }^{14,26,33}$ by having an essential role in the translocation of the damaged DNA to adjacent euchromatic regions where recombinational repair can be completed, safe from the complications associated with a high density of surrounding repetitive sequences. ${ }^{34}$

During meiosis, DSBs are initiated endogenously and are required for meiotic recombination and formation of chiasmata, which are essential for proper alignment and segregation of the chromosomes during the first meiotic division. ${ }^{35}$ However, meiotic recombination within the repetitive pericentromeric regions can lead to branched chromosome structures and meiotic segregation errors and should therefore be avoided. ${ }^{36}$ Indeed, more than 80 years ago it was discovered in Drosophila that the centromeres and the surrounding pericentromeric regions have relatively low rates of meiotic recombination. ${ }^{37}$ Numerous studies in various organisms, including human, subsequently confirmed this phenomenon ${ }^{36}$ and the centromeres and pericentromeric regions are now considered 'cold' spots with a significantly low rate of meiotic recombination. ${ }^{36}$ In yeast and Drosophila, meiotic recombination within pericentromeric regions is suppressed by formation of pericentromeric heterochromatin. ${ }^{38,39}$ Importantly, also during mouse meiosis, the centromeric regions are condensed in pericentromeric heterochromatin. ${ }^{40}$

Spermatogenesis, including spermatogonial differentiation, meiosis and subsequent spermiogenesis, ${ }^{41}$ is characterized by continuously ongoing, rapid and profound changes in composition and function of chromatin. Chromatin structure and dynamics safeguard genomic integrity, influence replication and transcription and steer the mitotic and meiotic cell cycles. Therefore, failure in the spatio-temporal organization of chromatin can cause genomic instability, which can lead to incorrect chromosome segregation, chromosomal aberrations and aneuploidy. During early spermatogenesis, failure to maintain genomic integrity can cause spermatogonial apoptosis, while later chromosomal misalignments can lead to infertility caused by meiotic arrest. ${ }^{42}$ Even though proper chromatin architecture sits at the fundament of efficient and safe reproduction, surprisingly little is known about the changing chromatin dynamics that are already clearly visible during spermatogonial differentiation. ${ }^{43,44}$

In mammals, Smc6 mRNA is predominately expressed in the testis ${ }^{45}$ and seems regulated by post-translational modifications. ${ }^{45,46}$ However, because knockout of Smc6 leads to embryonic lethality and mutation of its ATP hydrolysis motif has no apparent effect on fertility, ${ }^{47}$ its function during spermatogenesis remains to be elucidated. Here, we find that Smc6 functions as part of the pericentromeric heterochromatin domains that start to take shape when undifferentiated type $A$ spermatogonia (including the spermatogonial stem cells) become irreversibly committed toward meiosis and are referred to as differentiating spermatogonia. ${ }^{48}$ These domains persist in the subsequent intermediate and type B spermatogonia, are present during the entire process of meiosis and only disappear when haploid spermatids start to elongate. Interestingly, although not essential for spermatogonial mitosis, Smc6-negative meiotic cells often appear arrested in M-phase. We therefore propose that, in line with its function within heterochromatin in Drosophila, ${ }^{34}$ Smc6 has a key role in the suppression of meiotic recombination between and within centromeric regions and thus the prevention of accumulating branched chromosome structures that would otherwise cause meiotic arrest and, consequently, male infertility.

\section{Results}

Smc6 expression and localization in the testis. To investigate the presence of the Smc5/6 complex in the testis, we performed western blot analysis for Smc6 on several organs of the mouse, including the testis. Interestingly, Smc6 expression was much stronger in the testis than in other organs, showing a 20- to 100-fold increase in band-intensity compared with brain or other organs, respectively (Figure 1a; Supplementary Figure 1a). In contrast to a similar extra band observed in human cultured cells, ${ }^{45}$ we did not find the upper band for Smc6 to be caused by hyper-phosphorylation (Supplementary Figure 1b).

We next studied the localization of Smc6 in the different cell types present in the mouse testis by immunohistochemistry (Figure 1b). After examination of seminiferous tubules of all the stages of the seminiferous epithelium in four different animals, two subpopulations of type A spermatogonia were found; spermatogonia without nuclear Smc6 and spermatogonia showing an, often speckled, nuclear staining. This speckled staining pattern appeared stronger and more pronounced in the intermediate and type B spermatogonia. In spermatocytes, these speckles became larger and were most often located at the rim of the nucleus. In addition to the general faint cytoplasmic localization present in all germ cells, round spermatids showed Smc6 staining in the chromocenters present in the nucleus. In developing round spermatids, Smc6 was found to gradually decrease until only a faint cytoplasmic Smc6 staining remained in elongating spermatids. We did not detect Smc6 in any of the somatic cells of the testis, including the Sertoli cells, Leydig cells and peritubular cells.

Undifferentiated spermatogonia, including the spermatogonial stem cells, do not express Smc6. To further characterize the Smc6-negative and -positive spermatogonial subpopulations, we determined the number of Smc6-negative spermatogonia per 100 Sertoli cells in the different stages of the seminiferous epithelium (Figure 2a). 


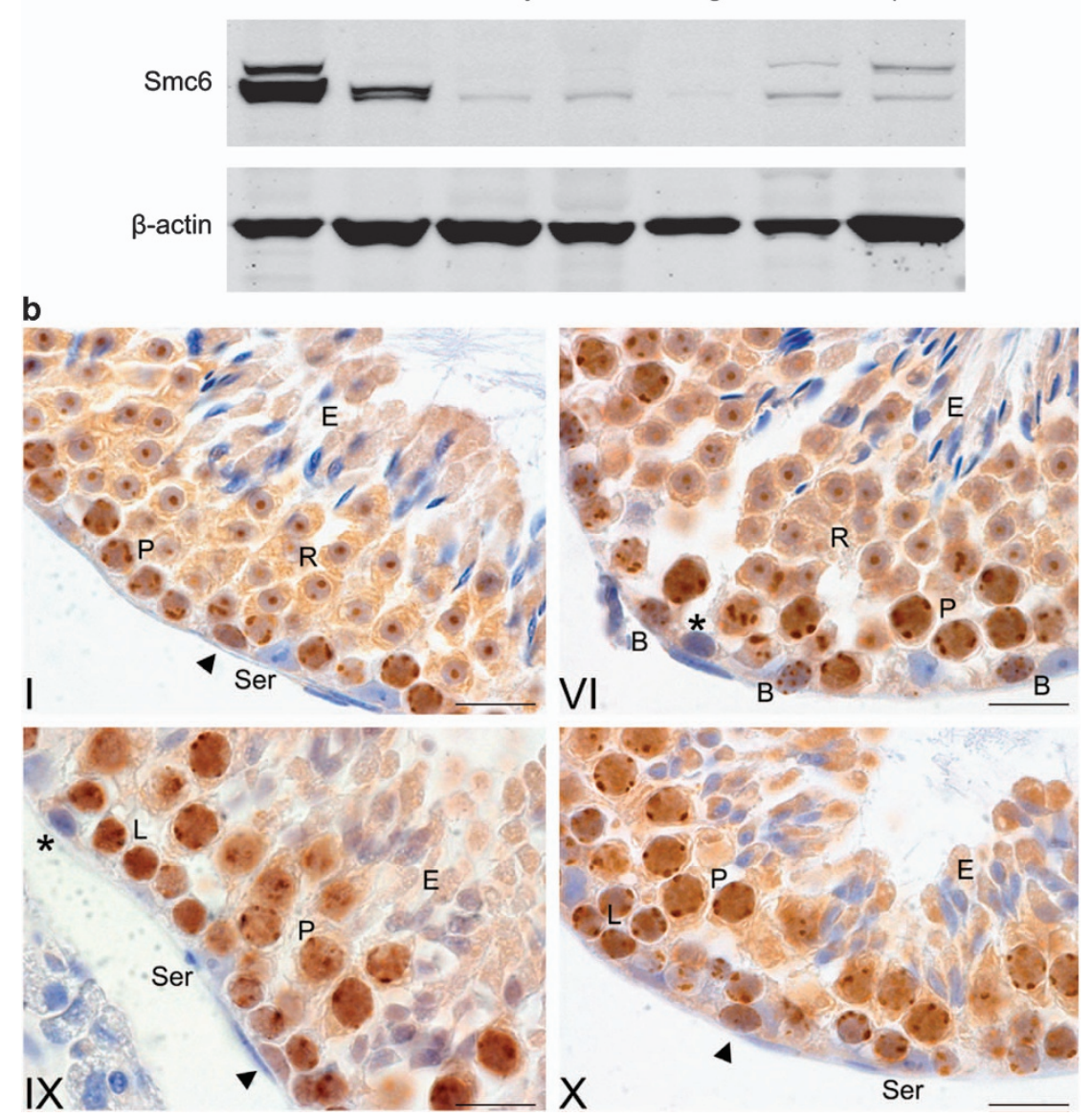

Figure 1 Characterization of Smc6 in the testis. (a) Western blot analysis of Smc6 in mouse organs. The intensity of the Smc6-bands in the testis was $>20$-fold higher compared with brain and $>100$-fold higher compared with the other organs studied, when corrected for the loading control ( $\beta$-actin). (b) Immunohistochemical localization of Smc6 in mouse testis sections. All epithelial stages were examined: depicted are stages I, VI, IX and X. Smc6 is only present in a subpopulation of type A spermatogonia, in all intermediate and B spermatogonia, spermatocytes and round spermatids. From stage IX and onwards, the elongating spermatids are negative for Smc6. Indicated are Smc6-positive (arrowheads) and -negative (asterisk) type A spermatogonia, type B spermatogonia $(B)$, pachytene $(P)$ and leptotene $(L)$ spermatocytes, round spermatids $(R)$ and elongated spermatids (E). Negative controls showed no staining. Bar $=20 \mu \mathrm{m}$

Interestingly, the observed quantitative pattern of Smc6-negative spermatogonia across the different epithelial stages matches with a similarly obtained pattern described for the presence of LIN28, an accepted marker for undifferentiated spermatogonia in the mouse. ${ }^{49}$ Accordingly, this pattern also indicates that the Smc6-positive type A spermatogonia are differentiating.

To verify that the Smc6 expression marks differentiating spermatogonia, we conducted immunohistochemistry for both Smc6 and LIN28 on consecutive sections of mouse testes. Using this approach, we found that when spermatogonia present in one section express Smc6, the same cells in a neighboring section are indeed negative for LIN28 (Figure 2b). Vice versa, spermatogonia that express LIN28 are negative for Smc6. Hence, undifferentiated spermatogonia are Smc6-negative and expression of Smc6 marks differentiating spermatogonia.

Smc6 expression coincides with induction of spermatogonial differentiation. To experimentally investigate whether Smc6 expression is directly correlated with the transition of undifferentiated to differentiating spermatogonia, we used a model of vitamin A-deficient (VAD) rats. Because retinoic acid, the active metabolite of vitamin $A$, is required for this transition, the only germ cells in the testes of these animals are undifferentiated spermatogonia. ${ }^{50}$ However, upon administration of retinoic acid, the arrested undifferentiated spermatogonia synchronously resume spermatogonial differentiation. ${ }^{50-52}$ In line with our previous experiments, all spermatogonia in the VAD testes were negative for Smc6 (Figure 2c). Only after retinoic acid administration, many Smc6-positive spermatogonia were found (Figure 2c), showing that the presence of Smc6 indeed marks spermatogonial differentiation and thus the transition after which spermatogonia are irreversibly committed to undergo meiosis and subsequent spermiogenesis.

Smc6 expression is localized in heterochromatin domains. Because the timing and expression pattern of Smc6 in differentiating spermatogonia appears similar to the gradual development of heterochromatin during spermatogenesis, ${ }^{43,44}$ we performed co-immunofluorescence 


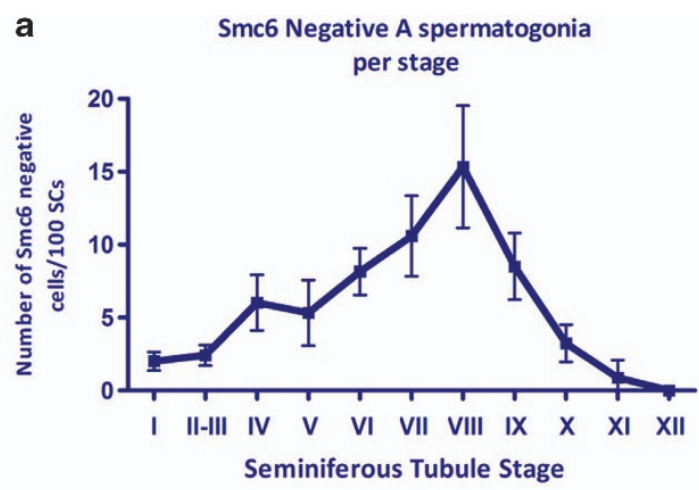

b

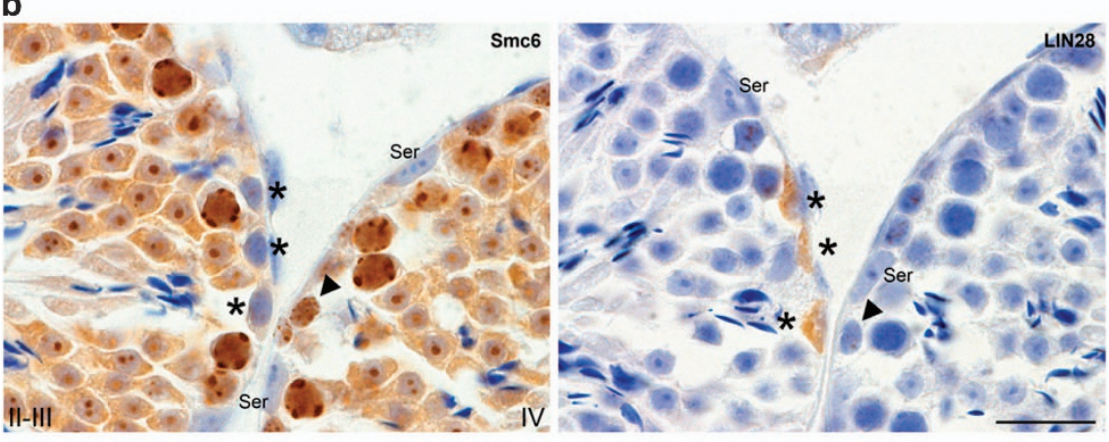

c
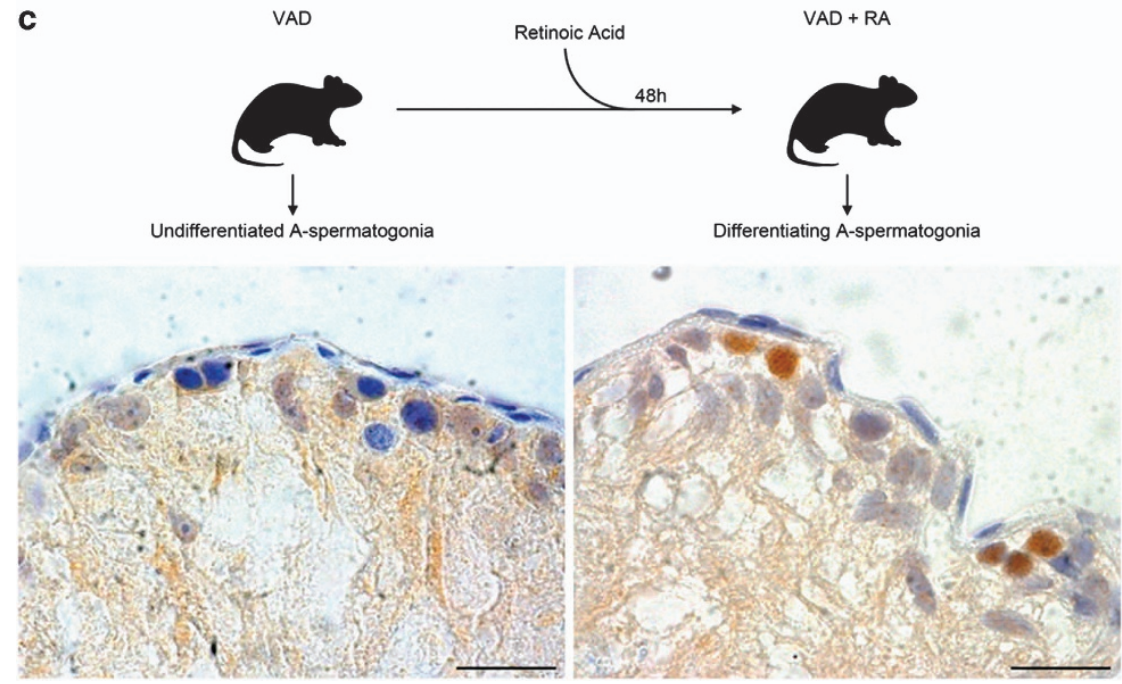

Figure 2 Smc6 is only expressed in differentiating spermatogonia. (a) The amount of Smc6-negative type A spermatogonia per 100 Sertoli cells counted per stage. For every epithelial stage, at least 10 tubules were counted; data are plotted as mean \pm S.E.M. (b) Consecutive sections of mouse testis were stained with anti-Smc6 or antiLIN28 and the same spermatogonia, when present in both sections, were compared. Smc6-negative cells (*) are LIN28-positive and vice versa (arrowheads). Epithelial stages are indicated with roman numerals (left panel). Sertoli cells are marked with Ser. (c) Testis sections from VAD rats, without and after administration of retinoic acid, were stained with anti-Smc6. Only after induction of differentiation by retinoic acid, Smc6-positive spermatogonia are found. Negative controls showed no staining. $\operatorname{Bar}=20 \mu \mathrm{m}$

microscopy on testis sections using antibodies against Smc6, the heterochromatin marker H3K9me3 and DAPI as DNA-stain to determine specific cell types by nuclear morphology and tubular context. In all Smc6-positive germ cells, nuclear Smc6 strongly localized to DAPI-bright regions that also contained H3K9me3. Hence, Smc6 is indeed enriched at sites of heterochromatin in differentiating spermatogonia, spermatocytes and round spermatids (Figure 3; Supplementary Figure 2).
Smc6 is always absent from the chromosomes in mitotic metaphase, whereas Smc6-negative spermatocytes do not complete the first meiotic division. As shown for mitotically dividing cells in culture, ${ }^{45}$ we find that Smc6 is never located on the chromosomes of mitotically dividing spermatogonia (Figure 4a). In contrast, Smc6 is strongly present on the chromosomes of all healthy meiotically dividing spermatocytes in what looks like centromeric regions or kinetochores (Figures $4 \mathrm{a}$ and b). Interestingly, of all 

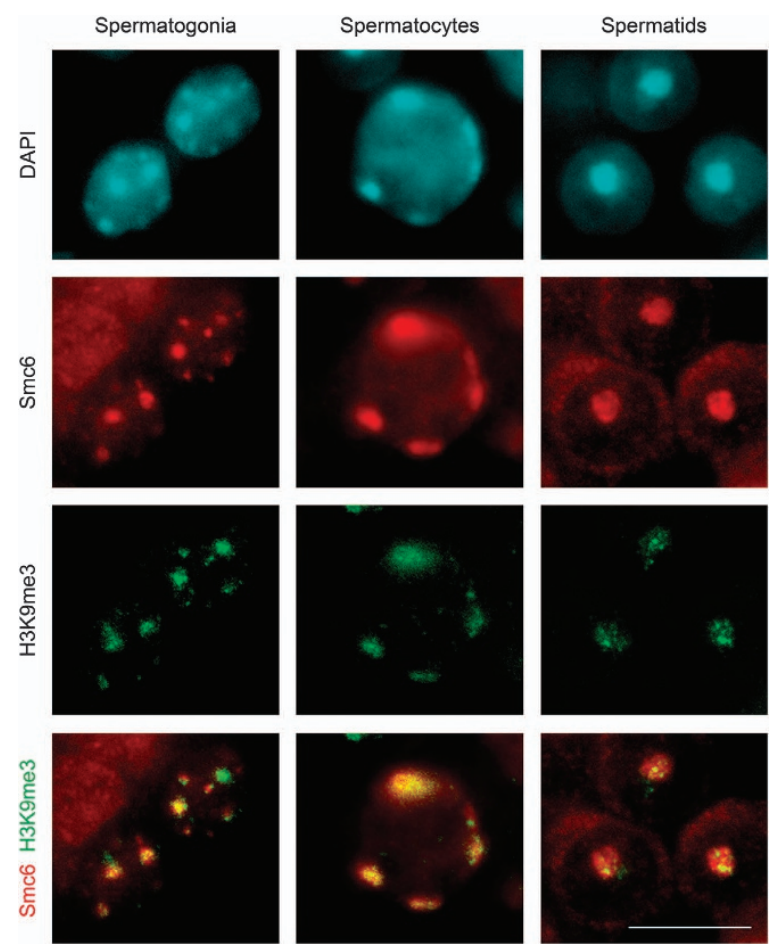

Figure 3 Smc6 localizes to heterochromatin domains. Co-immunofluorescent staining of testis sections for Smc6 and H3K9me3. Smc6 expression overlaps with heterochromatin, marked by H3K9me3, in all cell types expressing Smc6. DAPI was used for DNA counterstaining. Only negative controls for H3K9me3 showed staining in the interstitium and peritubular cells (Supplementary Figure S2). Bar $=10 \mu \mathrm{m}$

M-phase spermatocytes observed (434 cells examined in three different animals), $5.5 \%$ was found to be negative for Smc6. However, these Smc6-negative M-phase spermatocytes always appeared as single cells that were lagging behind and positioned between healthy synchronously dividing secondary spermatocytes or even round spermatids (Figures $4 \mathrm{c}$ and $\mathrm{d}$ ), indicating an inability to finish their first M-phase.

Smc6 is part of pericentromeric heterochromatin domains. To investigate possible roles for Smc6 during synaptonemal complex formation or meiotic recombination, we fluorescently stained meiotic spreads for Smc6 together with an antibody against the synaptonemal complex protein SCP3 and CREST-serum to mark the centromeres. Importantly, Smc6 appeared to be completely absent from the synaptonemal complex and never appeared as foci that could indicate a role in DNA repair or meiotic recombination. Instead, during all stages of the meiotic prophase, Smc6 co-localized exactly with DAPI-bright heterochromatic regions that surround the centromeres stained with CREST (Figure 5).

To find out whether Smc6-containing heterochromatin regions in non-meiotic germ cells also embed the centromeres, we performed co-immunofluorescence microscopy on testis section using an antibody against Smc6, CREST-serum and DAPI. Just as in meiotic spreads, brighter CREST dots co-localized with Smc6 in all Smc6-positive germ cells (Supplementary Figure S3). Hence, Smc6-containing pericentromeric heterochromatin domains already start to form during spermatogonial differentiation, continue to embed the centromeric regions during meiosis and gradually disappear in developing round spermatids.

Meiotic pericentromeric heterochromatin domains do not co-localize with DNA damage repair or recombination sites. To fully exclude a role for Smc6 during meiotic DSB repair, we first co-stained Smc6 with $\gamma \mathrm{H} 2 \mathrm{AX}$ in combination with either CREST or STAG3, to mark the centromeres or the synaptonemal complex, respectively (Figure 6). During early stages of meiosis, when the homologous chromosomes are not yet synapsed and more than 20 CREST dots are visible, $\gamma \mathrm{H} 2 \mathrm{AX}$ marks meiotic DSBs. ${ }^{53}$ However, in these cells, we did not observe co-localization between Smc6 and $\gamma \mathrm{H} 2 \mathrm{AX}$. Subsequently during pachytene, when the somatic chromosomes are fully synapsed, $\gamma \mathrm{H} 2 \mathrm{AX}$ only marks the $X Y$ body in which the largely unsynapsed $X$ and $Y$ chromosomes are silenced by meiotic sex chromosome inactivation $(\mathrm{MSCl}) .{ }^{54}$ In these cells, we did observe some overlap between $\gamma \mathrm{H} 2 \mathrm{AX}$ and Smc6, but mostly in the pericentromeric region containing a CREST dot. Occasionally, $\gamma \mathrm{H} 2 \mathrm{AX}$ also marks somatic chromosomes in pachytene-like cells, most likely due to aberrant synapsis causing meiotic silencing of unsynapsed chromosomes (MSUC). ${ }^{54}$ However, in such cells, Smc6 and $\gamma \mathrm{H} 2 \mathrm{AX}$ did not co-localize on the somatic chromosomes.

Most meiotic DSBs will be resolved by homologous recombination, which can be visualized by staining for Rad51. ${ }^{55}$ To investigate the spatial relation between pericentromeric heterochromatin and the presence of Rad51, we first co-labeled meiotic cells with Smc6 and H3K9me3 together with CREST or STAG3 to confirm pericentromeric heterochromatin domain structure and content in meiotic spread preparations (Figure 7a). We then used confocal microscopy to study whether Rad51 foci are localized to the pericentromeric heterochromatin domains, marked by H3K9me3. However, as shown in the max projection of the confocal layers (Figure $7 \mathrm{~b}$ ) or the supplemented $3 \mathrm{D}$ rendering of the same Z-scan (Supplementary Video 1), the meiotic pericentromeric heterochromatin domains did not co-localize with Rad51.

Taken together, these results show that during the first meiotic prophase, when synapsis between the homologous chromosomes and meiotic recombination take place, Smc6 is part of pericentromeric heterochromatin domains and does not have a role in early meiotic DSB repair, meiotic recombination or silencing of unsynapsed chromosomes.

\section{Discussion}

We identify a distinct Smc6-positive subpopulation of A spermatogonia that, based on its presence in specific spermatogenic stages and the lack of LIN28-expression, ${ }^{49}$ appears to represent differentiating spermatogonia. Unlike their undifferentiated progenitors, these spermatogonia are irreversibly committed to eventually enter meiosis and further divisions and development of these cells are strictly orchestrated by the stages of the seminiferous epithelium. ${ }^{41,48}$ To experimentally validate that Smc6 expression indeed 


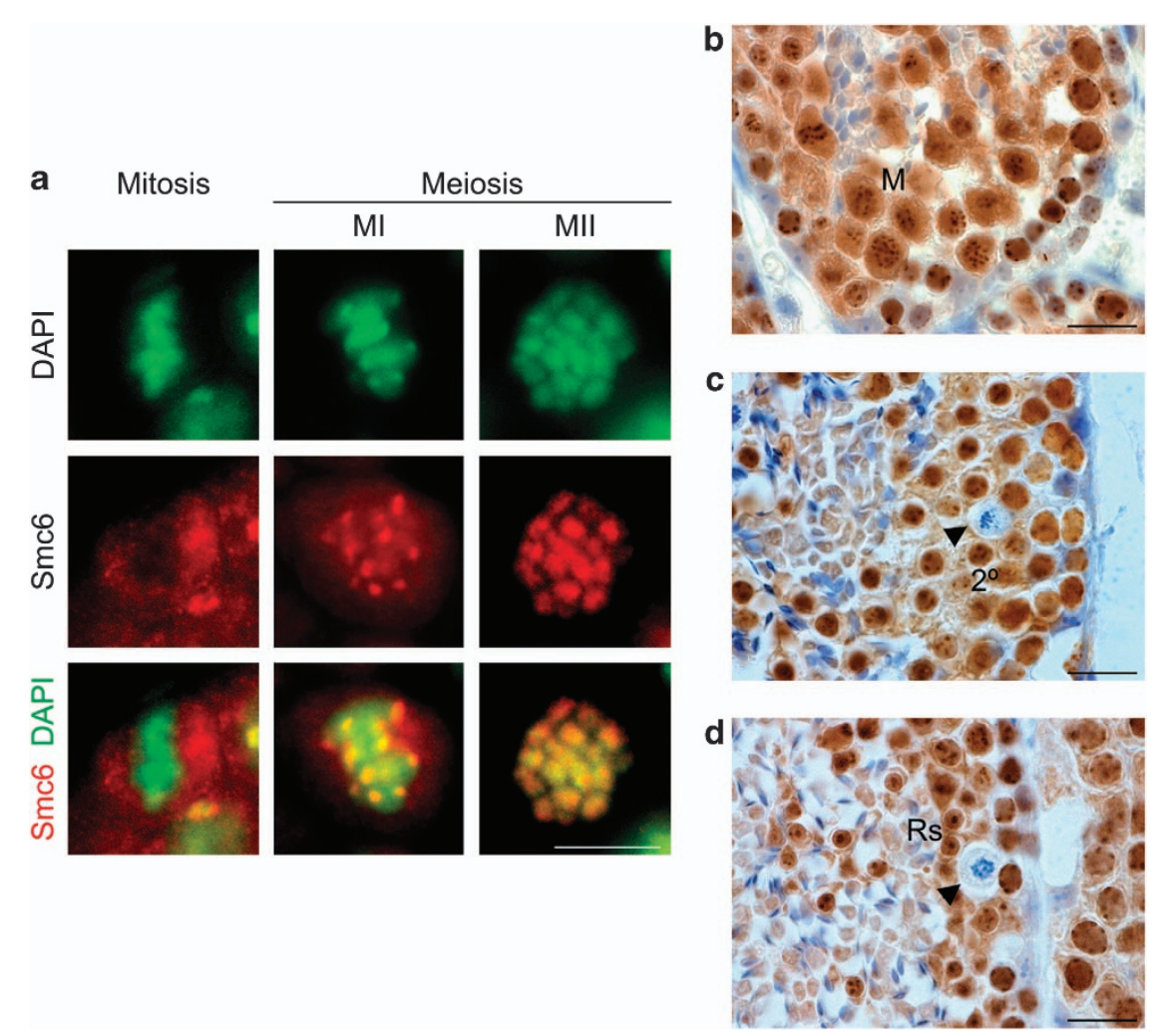

Figure 4 Smc6-negative M-phase spermatocytes arrest at the first meiotic division. (a) Fluorescent staining of testis sections for Smc6. Smc6 is located on the chromosomes (visualized by DAPI) during all healthy meiotic divisions but never during mitotic divisions. (b) Synchronously dividing Smc6-positive M-phase spermatocytes (M) in stage XII. (c) Still in stage XII, arrested Smc6-negative MI-phase spermatocyte (arrowhead) that did not proceed to secondary spermatocyte $\left(2^{\circ}\right)$. (d) Arrested Smc6-negative spermatocyte (arrowhead) present in stage I that should have proceeded to round spermatid (Rs). Negative controls showed no staining. Bar $=5 \mu \mathrm{m}$ (a) or $20 \mu \mathrm{m}(\mathbf{b}-\mathbf{d})$

coincides with spermatogonial differentiation, we used a VAD rat model in which arrested undifferentiated spermatogonia only commence spermatogonial differentiation upon retinoic acid administration. This model confirms that the Smc6positive spermatogonia are indeed differentiating. Still, because the germ cells present in the VAD rat model are all synchronized, one could argue that the observed change in Smc6 expression observed is cell cycle dependent. However, Smc6 protein expression has been shown to be cell cycle independent in cultured mammalian cells. ${ }^{45}$ Moreover, if Smc6 expression would depend on cell cycle phase, we would have found both Smc6-negative and -positive cells among the LIN28-expressing spermatogonia. Thus, our results convincingly show that Smc6 is excluded from the undifferentiated spermatogonia, including the spermatogonial stem cells, and specifically marks the differentiating spermatogonia.

Considering its spermatogonial expression pattern, Smc5/6 could be involved in the initiation of spermatogonial differentiation, analogous to the cohesin complex which, in combination with the chromatin binding protein CTCF, has been connected to chromatin changes that lead to altered gene expression. ${ }^{7-9}$ Moreover, independently of CTCF binding, cohesin co-localizes with pluripotency-related transcription factors in embryonic stem cells, thereby preventing differentiation. ${ }^{11}$ Interestingly, a testis-specific paralogue of CTCF (CTCF-like or CTCFL) exists that, despite strong similarities in DNA-binding consensus sequence, only shows minor overlap with CTCF in experimentally demonstrated DNA-binding sites. ${ }^{56,57}$ Moreover, where cohesion-binding sites largely co-localize with those of CTCF, ${ }^{11}$ they only co-localize with relatively few CTCFL-binding sites. ${ }^{57}$ Theoretically, if not cohesin, the Smc5/6 complex could be the SMC complex facilitating CTCFL-mediated gene regulation during spermatogenesis. In line with this hypothesis, it has been shown that CTCFL, as we find for Smc6, only starts to be expressed in late spermatogonia. ${ }^{57} \mathrm{~A}$ model in which the Smc5/6 complex functions together with CTCFL to regulate genes necessary for spermatogonial differentiation could therefore be appealing.

The differentiating spermatogonia are preparing their genome to undergo meiosis, a process that includes the induction of DSBs, synapsis of the homologous chromosomes, meiotic recombination and the subsequent meiotic divisions. ${ }^{41}$ The preparations for these events involve drastic changes in chromatin architecture, which in rodents, are illustrated by the increasing amount of heterochromatin that appears at the nuclear rim while the spermatogonia differentiate. ${ }^{43,44}$ As we have shown that Smc6 expression coincides with spermatogonial differentiation, we investigated whether Smc6 localization is also correlated with heterochromatin formation. By fluorescently co-staining testis sections for Smc6 and the heterochromatin marker H3K9me3, we demonstrated that Smc6 indeed co-localizes with heterochromatic regions. Additionally, by co-staining 

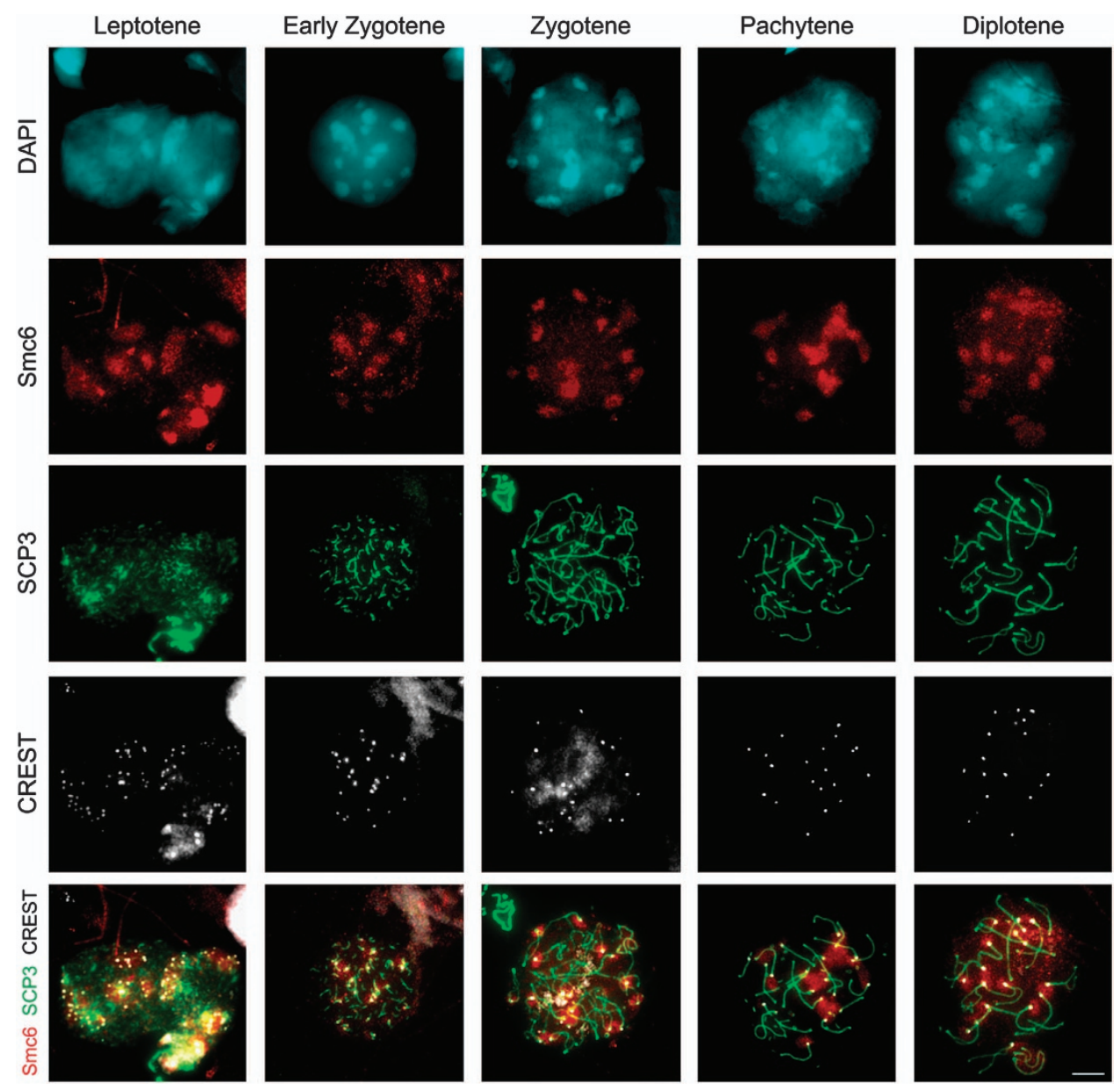

Figure 5 Smc6 is part of pericentromeric heterochromatin domains during meiosis. Co-immunofluorescent staining of meiotic spread preparations using antibodies against Smc6 and synaptonemal complex protein SCP3, CREST-serum to mark the centromeres and DAPI. Smc6 shows to be part of pericentromeric heterochromatin domains during all examined stages of the meiotic prophase. Negative controls showed no staining. Bar $=10 \mu \mathrm{m}$

testis sections for Smc6 and the centromere marker CREST, we show that these heterochromatic patches are actually pericentromeric heterochromatin domains that start to take shape during spermatogonial differentiation, continue throughout meiosis and early spermiogenesis and are characterized by the presence of Smc6.

In yeast, the Smc5/6 complex appeared essential to resolve meiotic recombination intermediates. ${ }^{15-17}$ During mammalian meiosis, DSBs are endogenously initiated (marked by $\gamma \mathrm{H} 2 \mathrm{AX}$ ) in early meiotic cells ${ }^{53}$ to be resolved later by homologous recombination (marked by Rad51). ${ }^{55}$ However, we observe that meiotic DSB sites marked by $\gamma \mathrm{H} 2 \mathrm{AX}$ or Rad51 do not co-localize with the Smc6/H3K9me3-positive pericentromeric heterochromatin domains. Moreover, as observed previously, ${ }^{45}$ we do not find Smc6 on the synaptonemal cores nor do we see any other Smc6 foci that would point to a function in meiotic recombination. Only in the $X Y$ body, in which $\gamma \mathrm{H} 2 \mathrm{AX}$ marks $\mathrm{MSCl}$ of the unsynapsed $X$ and $Y$ chromosomes, ${ }^{54}$ Smc6 and $\gamma \mathrm{H} 2 \mathrm{AX}$ partly overlap. However, this overlap is restricted to the pericentromeric region where the $\mathrm{X}$ and $\mathrm{Y}$ chromosomes are actually synapsed. Moreover, Smc6 and $\gamma \mathrm{H} 2 \mathrm{AX}$ do not co-localize in occasional pachytene-like cells that display $\gamma \mathrm{H} 2 \mathrm{AX}$ staining on somatic chromosomes. Because the presence of $\gamma \mathrm{H} 2 \mathrm{AX}$ indicates endogenously occurring synapsis problems in these cells, we do not consider Smc6 to directly contribute to MSUC.

Interestingly, also yeast-Smc6 is strongly enriched in the pericentromeric regions during the G2 phase of mitosis, which functionally resembles the prolonged first meiotic prophase, where it is essential for the timely separation of chromatids and the prevention of branched and entangled chromosome structures and subsequent mitotic arrest. ${ }^{26}$ An additional challenge that only meiotic cells face is that DSBs are endogenously induced and repaired by homologous recombination to generate genetic diversity among their haploid daughter cells. Because the pericentromeric regions are rich in repetitive sequences, they are prone to form intra-chromosomal recombination structures, which is why meiotic recombination around the centromeres is generally suppressed. ${ }^{36}$ However, the mechanism behind this centromere-specific suppression of meiotic recombination is currently not clear. In yeast and Drosophila, it has been shown that homologous recombination around the centromeres is suppressed by the formation of pericentromeric heterochromatin. ${ }^{38,39}$ Moreover, in Drosophila, Smc6 has been found to have a key role in the suppression of 

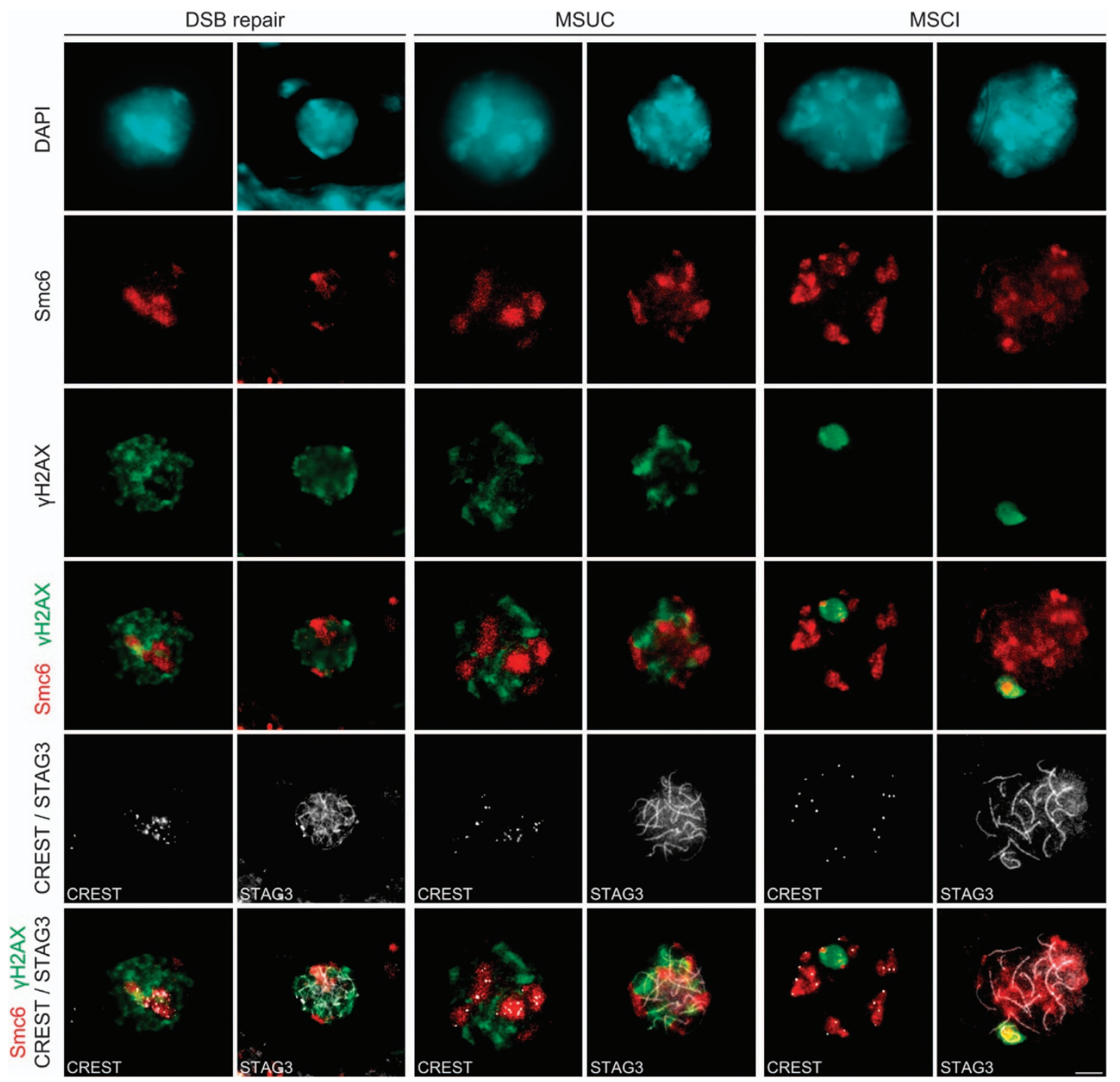

CREST

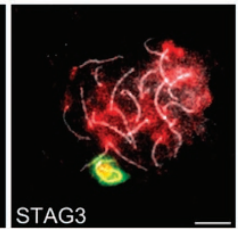

Figure 6 Meiotic Smc6 is not involved in $\gamma \mathrm{H} 2 \mathrm{AX}$-marked DSB repair or silencing of unsynapsed chromosomes. Co-immunofluorescent staining of meiotic spreads preparations using antibodies against Smc6, $\gamma$ H2AX and STAG3 (to mark the synaptonemal complex core), CREST-serum (to mark the centromeres) and DAPI. Smc6 shows no co-localization with $\gamma \mathrm{H} 2 \mathrm{AX}$ during early stages of meiosis when $\gamma \mathrm{H} 2 \mathrm{AX}$ marks DSBs (DSB repair) or in occasional pachytene-like cells representing MSUC. In healthy pachytene spermatocytes, when $\gamma \mathrm{H} 2 \mathrm{AX}$ only marks the $\mathrm{XY}$ body representing MSCl, Smc6 and $\gamma \mathrm{H} 2 \mathrm{AX}$ do overlap; mostly in the $\mathrm{XY}$ pericentromeric region marked by a CREST dot. Bar $=5 \mu \mathrm{m}$

homologous recombination in heterochromatin domains in somatic cells by inhibiting the recombinase Rad51 within these domains. ${ }^{34}$ As we find that Smc6 functions within pericentromeric heterochromatin domains over the entire time frame during which meiotic recombination takes place, we propose that Smc6 is involved in suppressing recombination within and between centromeric regions during mammalian meiosis. This hypothesis is strengthened by the observation that the meiotic pericentromeric heterochromatin domains indeed do not contain DSBs or meiotic recombination sites marked by $\gamma \mathrm{H} 2 \mathrm{AX}$ or Rad51, respectively.

We also found centromeric Smc6 expression during and in between the two subsequent meiotic divisions $\mathrm{MI}$ and MII. During yeast mitosis, Smc5/6 has been found to provide topological relief to replication forks ${ }^{4,21-28}$ and separating sister chromatids. ${ }^{26}$ Similarly, Smc6 could be involved in providing topological relief to the separating chromosomes during meiosis. Both centromeric recombination and topological stress will activate the spindle checkpoint and cause meiotic arrest. Indeed, Smc6-negative spermatocytes are arrested and thus mostly found in a subsequent stage in which the healthy spermatocytes have synchronously developed into round spermatids.

Even after completion of meiosis, Smc6 is still present in round spermatids within the chromocenter(s) in which the centromeres are located during spermiogenesis. However, Smc6 gradually disappears, as these haploid cells develop toward elongated spermatids. This Smc6 could be remnants of meiosis or could be involved in the subsequent pair-wise clustering and condensation of the centromeric regions in developing spermatids. ${ }^{40}$

To conclude, in this study we demonstrate roles for the chromatin-modifying protein Smc6 during spermatogonial differentiation, meiosis and perhaps even spermiogenesis. 
a
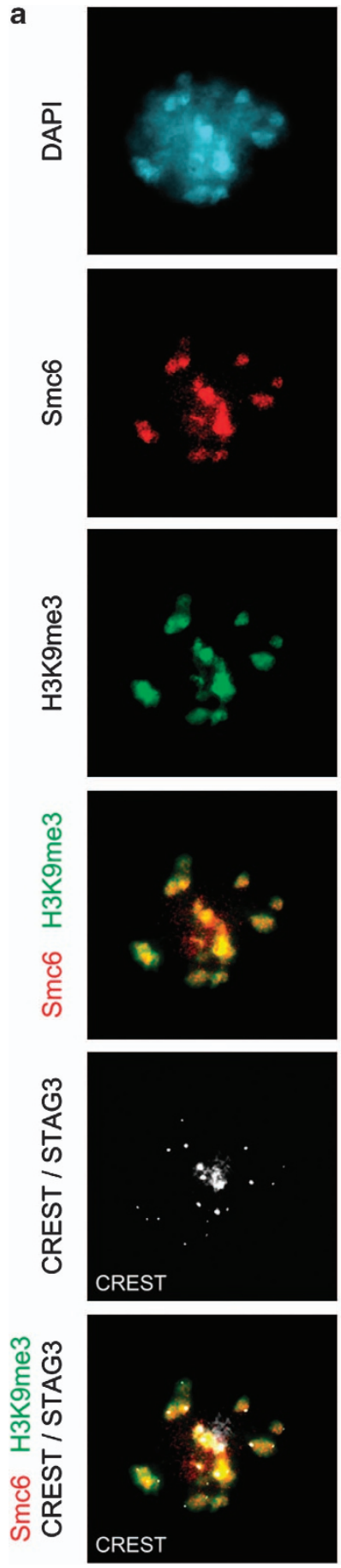
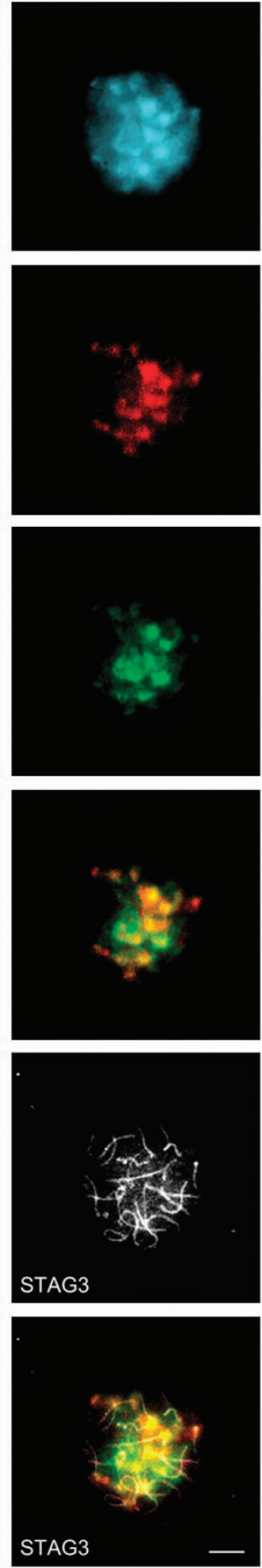

b
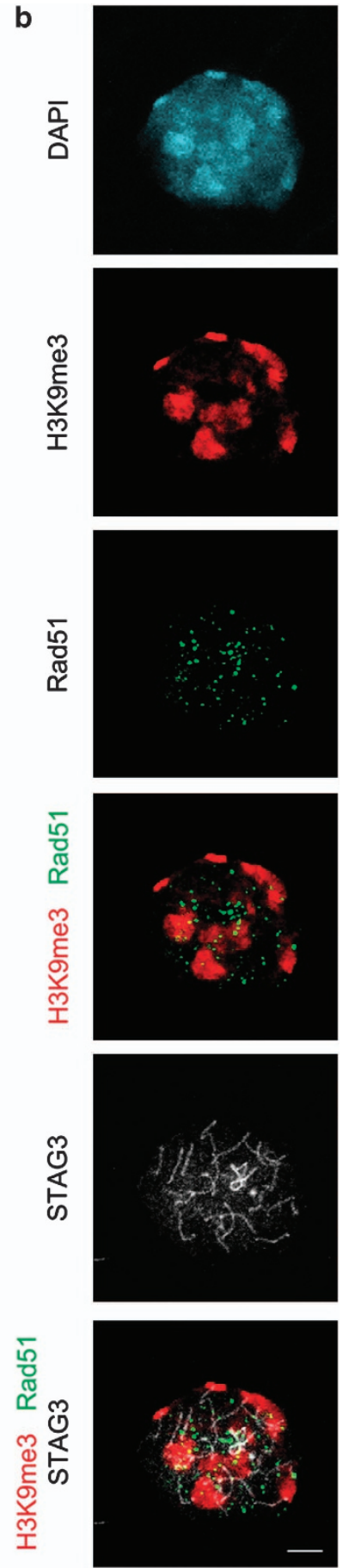

Figure 7 Meiotic Smc6 localizes to pericentromeric heterochromatin domains that are deprived of Rad51 foci. Co-immunofluorescent staining of meiotic spreads preparations using antibodies against Smc6, H3K9me3, Rad51 and STAG3 (to mark the synaptonemal complex core), CREST-serum (to mark the centromeres) and DAPI. (a) Smc6 co-localizes with pericentromeric heterochromatin marked by H3K9me3. (b) These pericentromeric heterochromatin show no Rad51 foci, indicating that meiotic recombination does not occur at these sites. Bar $=5 \mu \mathrm{m}$

Smc6 in differentiating spermatogonia could influence gene expression, possibly in cooperation with CTCFL. ${ }^{57}$ However, considering the fact that we find Smc6 to function in pericentromeric heterochromatin domains that already form in differentiating spermatogonia, Smc6 localization in these cells could also be part of chromatin modifications that prepare the cells for the upcoming meiotic events. During meiosis, recombination between and within the centromeric regions must be repressed to prevent the accumulation of branched chromosome structures that would otherwise cause meiotic arrest and, consequently, male infertility. This can be achieved by the formation of pericentromeric heterochromatin domains $^{38,39}$ and the presence of Smc6 within these domains to exclude Rad51-mediated recombination. ${ }^{34}$ We have now 
shown that the pericentromeric regions during mouse meiosis are indeed condensed in Smc6-positive/Rad51-negative heterochromatin domains. Moreover, we observe spermatocytes without Smc6 to be arrested, probably at the first meiotic division. Considering all these data, we hypothesize that, as in somatic heterochromatin in Drosophila, Smc6 has an important role in the suppression of recombination around the centromeres during mammalian germ cell development.

\begin{abstract}
Materials and Methods
Animals. Surplus adult male mice (strains 129, FVB and Balb-c, between 8 and 15 weeks old) were used for western blot analysis, immunohistochemistry and meiotic spreads. The animals were used and maintained according to regulations provided by the animal ethical committee of the Academic Medical Center, Amsterdam, which also approved the experiments. Previously obtained paraffin-embedded testes of VAD Wistar rats were used for immunohistochemistry. ${ }^{50}$ All experiments were repeated using at least four different animals.
\end{abstract}

Western blotting. Pieces of mouse organ tissue $(4 \times 6 \mathrm{~mm})$ were placed in RIPA buffer ( $1 \%$ NP40, $0.5 \%$ sodium deoxycholate, $0.1 \%$ SDS) containing PMSF $(100 \mu \mathrm{M})$, protease inhibitor cocktail (PIC, $1 \times$, Roche, Almere, The Netherlands) and PhosSTOP (Roche). Tissues were homogenized and lysed on ice for $1 \mathrm{~h}$. After centrifugation ( $15 \mathrm{~min} / 14000$ r.p.m. $\left./ 4^{\circ} \mathrm{C}\right)$, the supernatant was collected and stored at $-80^{\circ} \mathrm{C}$ for a minimum of $1 \mathrm{~h}$. Protein concentrations were measured using the Qubit Protein assay Kit (Molecular Probes, Invitrogen, Carlsbad, CA, USA). Of each sample, $30 \mu \mathrm{g}$ was loaded on a $4-12 \%$ gradient gel (Invitrogen). Proteins were blotted on an Immobilon-FL PVDF membrane (Millipore, Billerica, MA, USA) and blocked with Odyssey Blocking buffer (LI-COR Biosciences, Westburg, Leusden, The Netherlands) for $1 \mathrm{~h}$ at room temperature. Thereafter, the membranes were incubated $\mathrm{o} / \mathrm{n}$ at $4^{\circ} \mathrm{C}$ in the primary antibody rabbit anti-Smc6 1:5000 (ab18039, Abcam, Cambridge, UK), diluted in Odyssey Blocking buffer containing $0.1 \%$ Tween-20. After washing three times for $10 \mathrm{~min}$ in TBST (Tris buffered saline containing $0.1 \%$ Tween-20), membranes were incubated in an IRDye $800 \mathrm{CW}$ secondary antibody $1: 15000$ for $1 \mathrm{~h}$ at room temperature (diluted in Odyssey Blocking buffer containing $0.1 \%$ Tween-20). As a loading control, a primary mouse anti- $\beta$-actin antibody 1:5000 (A1978, Sigma-Aldrich, St. Louis, MO, USA) was used. Image acquisition and quantification were done with the Odyssey Infrared Imaging system (LI-COR Biosciences). To determine phosphorylation of Smc6, samples were lysed in RIPA buffer with and without PhosSTOP. Samples lacking phosphatase inhibitors were treated with Calf Intestinal alkaline phosphatase (New England Biolabs, Leiden, The Netherlands) for $1 \mathrm{~h}$ at $37^{\circ} \mathrm{C}$, after which the samples were processed as described.

Immunohistochemistry. Immunohistochemical staining was performed on $5-\mu \mathrm{m}$ mouse testis sections, previously fixed in $4 \%$ formaldehyde and embedded in paraffin. Antigen retrieval was performed on deparaffinated sections in sodium citrate, $\mathrm{pH} 6.0$ at $98^{\circ} \mathrm{C}$ for $10 \mathrm{~min}$. Nonspecific adhesion sites were blocked for $1 \mathrm{~h}$ at room temperature in PBST (phosphate-buffered saline containing $0.1 \%$ Tween-20) containing $10 \%$ goat serum.

To visualize Smc6 and LIN28, sections were subsequently incubated with the primary rabbit anti-Smc6 antibody 1:500 (ab18039, Abcam) or the primary rabbit anti-LIN28 antibody 1:5000 (ab46020, Abcam) for $2 \mathrm{~h}$ at room temperature. After washing sufficiently in PBS, the sections were incubated in the biotinylated secondary antibody goat-anti-rabbit. Signal was visualized using the avidin-biotin complex method: 60 min incubation with the pre-incubated $A B C$ solution (containing avidin and biotinylated horseradish peroxidase, Vector Laboratories, Burlingame, CA, USA) followed by $3,3^{\prime}$-diaminobenzidine as a substrate and counterstained with haematoxylin. For negative controls, isotype rabbit IgG was used instead of the primary antibody in corresponding concentrations. Slides were examined and pictures were taken using an Olympus BX41 bright-field microscope with an Olympus DP20 color camera.

To visualize $\mathrm{Smc6}$ with $\mathrm{H} 3 \mathrm{H} 9 \mathrm{me} 3$ and/or CREST, the sections were incubated $\mathrm{o} / \mathrm{n}$ at $4{ }^{\circ} \mathrm{C}$ with rabbit anti-Smc6 1:500 (ab18039, Abcam) and mouse anti-H3K9me3 antibody 1:200 (ab6001, Abcam) or human CREST-serum 1:500 (90C-CS1058, FitzGerald, Acton, MA, USA). Proteins were visualized using fluorescently labeled secondary antibodies (donkey anti-rabbit Alexa Fluor 555 , goat anti-mouse Alexa Fluor 488 and donkey anti-human Alexa Fluor 647 respectively
(Invitrogen). DAPI was used as a nuclear counterstaining. For negative controls, sections were incubated without primary antibodies. Section were mounted in ProLong Gold (Invitrogen) and fluorescence microscopy images were acquired at room temperature using a microscope (DM RA HC; Leica) equipped with a Plan Apo $100 \times 1.40$ oil objective (Carl Zeiss, Inc., Sliedrecht, The Netherlands) and a cooled charge-coupled device camera (KX1400; Apogee Instruments, Logan, UT, USA). Images were acquired and analyzed using custom-made and Image-Pro Plus software. The presented figures were constructed using Adobe Photoshop CS5 version 12.0 .

Meiotic spreads. Cryopreserved testes from adult Balb-c mice were thawed and meiotic spreads were made using the 'drying-down' technique described by Peters et al. ${ }^{58}$ Staining protocol and image acquisition was identical to the immunohistochemical staining described above using rabbit anti-Smc6, human CREST-serum, mouse anti-SCP3 1:500 (ab97672, Abcam), mouse anti- $\gamma \mathrm{H} 2 \mathrm{AX}$ 1:10000 (05-636, Millipore), guinea pig anti-STAG3 1:100 (kindly provided by Anna Kouznetsova and Christer Höög), mouse anti-H3K9me3 1:500 (ab71604, Abcam) and rabbit anti-Rad51 1:100 (kindly provided by Jan Stap). ${ }^{59}$ For fluorescent labeling, additional secondary antibodies were used: goat anti-mouse Alexa Fluor 555, goat anti-guinea pig Alexa Fluor 488 and goat-anti-rabbit Alexa Fluor 647 (Invitrogen). Image acquisition and analysis was performed as described above. For Figure $7 \mathrm{~b}$ and Supplementary Video 1, confocal microscopy was performed using a Leica TCS SP8 SMD, mounted on a Leica DMI6000 inverted microscope. A 405-nm UV diode and 470-670 nm White Light Laser were used for excitation, and PMT and HyD detectors for detection. Images were obtained using a HC Plan Apo $63 \times 1.40$ oil objective and were analyzed using Leica Application Suite Advanced Fluorescence software.

\section{Conflict of Interest}

The authors declare no conflict of interest.

Acknowledgements. We thank Jan Stap, Daisy Picavet and the van Leeuwenhoek Centre for Advanced Microscopy-AMC for assistance with fluorescence imaging and confocal microscopy, use of their equipment and providing the antibody against Rad51 ${ }^{59}$ we thank Anna Kouznetsova and Christer Höög for sharing their antibody against STAG3. This study has been supported by an AMC Fellowship and The People Programme (Marie Curie Actions) of the European Union's Seventh Framework Programme (CIG 293765).

1. Nasmyth $\mathrm{K}$, Haering $\mathrm{CH}$. The structure and function of SMC and kleisin complexes. Annu Rev Biochem 2005; 74: 595-648.

2. Peters JM, Tedeschi A, Schmitz J. The cohesin complex and its roles in chromosome biology. Genes Dev 2008; 22: 3089-3114.

3. Bauerschmidt C, Woodcock M, Stevens DL, Hill MA, Rothkamm K, Helleday T. Cohesin phosphorylation and mobility of SMC1 at ionizing radiation-induced DNA double-strand breaks in human cells. Exp Cell Res 2011; 317: 330-337.

4. Wu N, Yu H. The Smc complexes in DNA damage response. Cell Biosci 2012; 2: 5.

5. Hudson DF, Marshall KM, Earnshaw WC. Condensin: architect of mitotic chromosomes. Chromosome Res 2009; 17: 131-144.

6. Wood AJ, Severson AF, Meyer BJ. Condensin and cohesin complexity: the expanding repertoire of functions. Nat Rev Genet 2010; 11: 391-404.

7. Parelho V, Hadjur S, Spivakov M, Leleu M, Sauer S, Gregson HC et al. Cohesins functionally associate with CTCF on mammalian chromosome arms. Cell 2008; 132: 422-433.

8. Wendt KS, Yoshida K, Itoh T, Bando M, Koch B, Schirghuber E et al. Cohesin mediates transcriptional insulation by CCCTC-binding factor. Nature 2008; 451: 796-801.

9. Rubio ED, Reiss DJ, Welcsh PL, Disteche CM, Filippova GN, Baliga NS et al. CTCF physically links cohesin to chromatin. Proc Natl Acad Sci USA 2008; 105: 8309-8314.

10. Hou C, Dale R, Dean A. Cell type specificity of chromatin organization mediated by CTCF and cohesin. Proc Natl Acad Sci USA 2010; 107: 3651-3656.

11. Nitzsche A, Paszkowski-Rogacz M, Matarese F, Janssen-Megens EM, Hubner NC, Schulz H et al. RAD21 cooperates with pluripotency transcription factors in the maintenance of embryonic stem cell identity. PLoS One 2011; 6: e19470.

12. De Piccoli G, Cortes-Ledesma F, Ira G, Torres-Rosell J, Uhle S, Farmer S et al. Smc5-Smc6 mediate DNA double-strand-break repair by promoting sister-chromatid recombination. Nat Cell Biol 2006; 8: 1032-1034.

13. Potts PR, Porteus MH, Yu H. Human SMC5/6 complex promotes sister chromatid homologous recombination by recruiting the $\mathrm{SMC} 1 / 3$ cohesin complex to double-strand breaks. EMBO J 2006; 25: 3377-3388. 
14. Potts PR. The Yin and Yang of the MMS21-SMC5/6 SUMO ligase complex in homologous recombination. DNA Repair 2009; 8: 499-506.

15. Wehrkamp-Richter S, Hyppa RW, Prudden J, Smith GR, Boddy MN. Meiotic DNA joint molecule resolution depends on Nse5-Nse6 of the Smc5-Smc6 holocomplex. Nucleic Acids Res 2012; 40: 9633-9646.

16. Farmer $\mathrm{S}$, San-Segundo PA, Aragón L. The smc5-smc6 complex is required to remove chromosome junctions in meiosis. PLoS One 2011; 6: e20948.

17. Pebernard S, McDonald WH, Pavlova Y, Yates JR, Boddy MN. Nse1, Nse2, and a novel subunit of the Smc5-Smc6 complex, Nse3, play a crucial role in meiosis. Mol Biol Cell 2004; 15: $4866-4876$

18. Bryan TM, Englezou A, Dalla-Pozza L, Dunham MA, Reddel RR. Evidence for an alternative mechanism for maintaining telomere length in human tumors and tumor-derived cell lines. Nat Med 1997; 3: 1271-1274.

19. Potts PR, Yu H. The SMC5/6 complex maintains telomere length in ALT cancer cells through SUMOylation of telomere-binding proteins. Nat Struct Mol Biol 2007; 14: 581-590.

20. Dunham MA, Neumann AA, Fasching CL, Reddel RR. Telomere maintenance by recombination in human cells. Nat Genet 2000; 26: 447-450.

21. De Piccoli G, Torres-Rosell J, Aragon L. The unnamed complex: what do we know about Smc5-Smc6? Chromosome Res 2009; 17: 251-263.

22. Bustard DE, Menolfi D, Jeppsson K, Ball LG, Dewey SC, Shirahige K et al. During replication stress, non-SMC element 5 (NSE5) is required for Smc5/6 protein complex functionality at stalled forks. J Biol Chem 2012; 287: 11374-11383.

23. Irmisch A, Ampatzidou E, Mizuno K, O'Connell MJ, Murray JM. Smc5/6 maintains stalled replication forks in a recombination-competent conformation. EMBO J 2009; 28: 144-155.

24. Kegel A, Betts-Lindroos H, Kanno T, Jeppsson K, Strom L, Katou Y et al. Chromosome length influences replication-induced topological stress. Nature 2011; 471: 392-396.

25. Ampatzidou E, Irmisch A, O'Connell MJ, Murray JM. Smc5/6 is required for repair at collapsed replication forks. Mol Cell Biol 2006; 26: 9387-9401.

26. Lindroos HB, Strom L, Itoh T, Katou Y, Shirahige K, Sjogren C. Chromosomal association of the $\mathrm{Smc5} / 6$ complex reveals that it functions in differently regulated pathways. $\mathrm{Mol} \mathrm{Cell}$ 2006; 22: 755-767.

27. Branzei D, Sollier J, Liberi G, Zhao X, Maeda D, Seki M et al. Ubc9- and mms21-mediated sumoylation counteracts recombinogenic events at damaged replication forks. Cell 2006; 127: 509-522.

28. Kegel A, Sjögren C. The Smc5/6 complex: more than repair? Cold Spring Harb Symp Quant Biol 2010; 75: 179-187.

29. Cuperus G, Shore D. Restoration of silencing in Saccharomyces cerevisiae by tethering of a novel Sir2-interacting protein, Esc8. Genetics 2002; 162: 633-645.

30. Dhillon N, Kamakaka RT. A histone variant, Htz1p, and a Sir1p-like protein, Esc2p, mediate silencing at HMR. Mol Cell 2000; 6: 769-780.

31. Yu Q, Kuzmiak H, Olsen L, Kulkarni A, Fink E, Zou Y et al. Saccharomyces cerevisiae Esc2p interacts with Sir2p through a small ubiquitin-like modifier (SUMO)-binding motif and regulates transcriptionally silent chromatin in a locus-dependent manner. J Biol Chem 2010; 285: 7525-7536.

32. Zhao X, Blobel GA. SUMO ligase is part of a nuclear multiprotein complex that affects DNA repair and chromosomal organization. Proc Natl Acad Sci USA 2005; 102 4777-4782.

33. Torres-Rosell J, Sunjevaric I, De Piccoli G, Sacher M, Eckert-Boulet N, Reid R et al. The Smc5-Smc6 complex and SUMO modification of Rad52 regulates recombinational repair at the ribosomal gene locus. Nat Cell Biol 2007; 9: 923-931.

34. Chiolo I, Minoda A, Colmenares SU, Polyzos A, Costes SV, Karpen GH. Double-strand breaks in heterochromatin move outside of a dynamic HP1a domain to complete recombinational repair. Cell 2011; 144: 732-744.

35. Petronczki M, Siomos MF, Nasmyth K. Un ménage à quatre: the molecular biology of chromosome segregation in meiosis. Cell 2003; 112: 423-440.

36. Lynn A, Ashley T, Hassold T. Variation in human meiotic recombination. Annu Rev Genomics Hum Genet 2004; 5: 317-349.

37. Beadle GWA. Possible influence of the spindle fibre on crossing-over in Drosophila. Proc Natl Acad Sci USA 1932; 18: 160-165.

38. Ellermeier C, Higuchi EC, Phadnis N, Holm L, Geelhood JL, Thon G et al. RNAi and heterochromatin repress centromeric meiotic recombination. Proc Natl Acad Sci USA 2010; 107: 8701-8705

39. Westphal T, Reuter G. Recombinogenic effects of suppressors of position-effect variegation in Drosophila. Genetics 2002; 160: 609-621.
40. Brinkley BR, Brenner SL, Hall JM, Tousson A, Balczon RD, Valdivia MM. Arrangements of kinetochores in mouse cells during meiosis and spermiogenesis. Chromosoma 1986; 94 : 309-317.

41. Jan SZ, Hamer G, Repping S, de Rooij DG, van Pelt AM, Vormer TL. Molecular control of rodent spermatogenesis. Biochim Biophys Acta 2012; 1822: 1838-1850.

42. de Rooij DG, de Boer P. Specific arrests of spermatogenesis in genetically modified and mutant mice. Cytogenet Genome Res 2003; 103: 267-276.

43. Chiarini-Garcia H, Russell LD. High-resolution light microscopic characterization of mouse spermatogonia. Biol Reprod 2001; 65: 1170-1178.

44. Chiarini-Garcia H, Russell LD. Characterization of mouse spermatogonia by transmission electron microscopy. Reproduction 2002; 123: 567-577.

45. Taylor EM, Moghraby JS, Lees JH, Smit B, Moens PB, Lehmann AR. Characterization of a novel human SMC heterodimer homologous to the Schizosaccharomyces pombe Rad18/Spr18 complex. Mol Biol Cell 2001; 12: 1583-1594.

46. Taylor EM, Copsey AC, Hudson JJR, Vidot S, Lehmann AR. Identification of the proteins, including MAGEG1, that make up the human SMC5-6 protein complex. Mol Cell Biol 2008; 28: $1197-1206$

47. Ju L, Wing J, Taylor E, Brandt R, Slijepcevic P, Horsch M et al. SMC6 is an essential gene in mice, but a hypomorphic mutant in the ATPase domain has a mild phenotype with a range of subtle abnormalities. DNA Repair 2013; 12: 356-366.

48. de Rooij DG, Russell LD. All you wanted to know about spermatogonia but were afraid to ask. J Androl 2000; 21: 776-798.

49. Zheng $\mathrm{K}$, Wu X, Kaestner KH, Wang PJ. The pluripotency factor LIN28 marks undifferentiated spermatogonia in mouse. BMC Dev Biol 2009; 9: 38.

50. van Pelt AM, de Rooij DG. Retinoic acid is able to reinitiate spermatogenesis in vitamin A-deficient rats and high replicate doses support the full development of spermatogenic cells. Endocrinology 1991; 128: 697-704

51. van Pelt AM, de Rooij DG. Synchronization of the seminiferous epithelium after vitamin A replacement in vitamin A-deficient mice. Biol Reprod 1990; 43: 363-367.

52. Van Pelt AM, De Rooij DG. The origin of the synchronization of the seminiferous epithelium in vitamin A-deficient rats after vitamin A replacement. Biol Reprod 1990; 42: 677-682.

53. Mahadevaiah SK, Turner JM, Baudat F, Rogakou EP, de Boer P, Blanco-Rodriguez J et al. Recombinational DNA double-strand breaks in mice precede synapsis. Nat Genet 2001; 27: $271-276$

54. Turner JMA, Mahadevaiah SK, Fernandez-Capetillo O, Nussenzweig A, Xu X, Deng C-X et al. Silencing of unsynapsed meiotic chromosomes in the mouse. Nat Genet 2005; 37: 41-47.

55. Moens PB, Kolas NK, Tarsounas M, Marcon E, Cohen PE, Spyropoulos B. The time course and chromosomal localization of recombination-related proteins at meiosis in the mouse are compatible with models that can resolve the early DNA-DNA interactions without reciprocal recombination. J Cell Sci 2002; 115: 1611-1622.

56. Loukinov DI, Pugacheva E, Vatolin S, Pack SD, Moon H, Chernukhin I et al. BORIS, a novel male germ-line-specific protein associated with epigenetic reprogramming events, shares the same 11-zinc-finger domain with CTCF, the insulator protein involved in reading imprinting marks in the soma. Proc Natl Acad Sci USA 2002; 99: $6806-6811$

57. Sleutels F, Soochit W, Bartkuhn M, Heath H, Dienstbach S, Bergmaier P et al. The male germ cell gene regulator CTCFL is functionally different from CTCF and binds CTCF-like consensus sites in a nucleosome composition-dependent manner. Epigenetics Chromatin 2012; 5 : 8.

58. Peters AH, Plug AW, van Vugt MJ, de Boer P. A drying-down technique for the spreading of mammalian meiocytes from the male and female germline. Chromosome Res 1997; 5 : 66-68

59. van Veelen LR, Cervelli T, van de Rakt MW, Theil AF, Essers J, Kanaar R. Analysis of ionizing radiation-induced foci of DNA damage repair proteins. Mutat Res 2005; 574 : 22-33

(2) (1) $\odot$ Cell Death and Disease is an open-access journal published by Nature Publishing Group. This work is licensed under a Creative Commons Attribution-NonCommercialNoDerivs 3.0 Unported License. To view a copy of this license, visit http://creativecommons.org/licenses/by-nc-nd/3.0/ 